\title{
Integration of Linear Dynamic Emission and Climate Models with Air Traffic Simulations
}

\author{
Banavar Sridhar ${ }^{1}$ \\ NASA Ames Research Center, Moffett Field, CA 94035-1000 \\ Hok K. $\mathrm{Ng}^{2}$ \\ University of California, Santa Cruz, Moffett Field, CA 94035-1000 \\ and \\ Neil Y. Chen ${ }^{3}$ \\ NASA Ames Research Center, Moffett Field, CA 94035-1000
}

\begin{abstract}
Future air traffic management systems are required to balance the conflicting objectives of maximizing safety and efficiency of traffic flows while minimizing the climate impact of aviation emissions and contrails. Integrating emission and climate models together with air traffic simulations improve the understanding of the complex interaction between the physical climate system, carbon and other greenhouse gas emissions and aviation activity. This paper integrates a national-level air traffic simulation and optimization capability with simple climate models and carbon cycle models, and climate metrics to assess the impact of aviation on climate. The capability can be used to make trade-offs between extra fuel cost and reduction in global surface temperature change. The parameters in the simulation can be used to evaluate the effect of various uncertainties in emission models and contrails and the impact of different decision horizons. Alternatively, the optimization results from the simulation can be used as inputs to other tools that monetize global climate impacts like the FAA's Aviation Environmental Portfolio Management Tool for Impacts.
\end{abstract}

\section{Introduction}

The development and evaluation of concepts and technology to support future air traffic management systems require a hierarchy of models ranging from real-time simulations to extensive field evaluations. Air traffic simulation models such as Airspace Concept Evaluation System (ACES) ${ }^{1}$, Center Tracon Automation System $(\mathrm{CTAS})^{2}$, Future Air traffic management Concept Evaluation Tool (FACET) ${ }^{3}$ and others are used to design air traffic systems balancing the conflicting objectives of maximizing safety, meeting future demands for airports and airspace and increase efficiency of traffic flows in the presence of uncertain weather. The impact of aviation emissions and contrails on climate imposes another constraint on the design of aircraft and aviation operations. The understanding of the complex interaction between the physical climate system, carbon and other greenhouse gas emissions and aviation activity can be improved by the development of integrated assessment models that include emission and climate models together with air traffic simulations.

The impact of various greenhouse gas emissions on the climate depends on the amount of emission of each type of gas, the residual amount of gas in the atmosphere and the lifetime of the gas. The concentration of $\mathrm{CO}_{2}$ in the atmosphere today, 390 parts per million ( $\mathrm{ppm}$ ), is far above the natural range of 172 to $300 \mathrm{ppm}$ observed before the 20th century and the increase is attributed to man-made activities ${ }^{4} . \mathrm{CO}_{2}$ is the most important anthropogenic greenhouse gas and its impact on the climate as measured by radiative forcing is larger than all other emissions such as methane, nitrous oxide, ozone and water vapor. It is estimated that aviation is responsible for $2 \%$ of all anthropogenic $\mathrm{CO}_{2}$ emissions. Oxides of nitrogen, commonly referred to as $\mathrm{NOx}$, created by the high temperatures

\footnotetext{
${ }^{1}$ Senior Scientist for Air Transportation Systems, Aviation Systems Division, Fellow.

${ }^{2}$ Senior Software Engineer, U.C. Santa Cruz, MS 210-8, Member AIAA.

${ }^{3}$ Aerospace Research Engineer, Systems Modeling and Optimization Branch, MS 210-10, Member AIAA.
}

1

American Institute of Aeronautics and Astronautics 
in the aircraft engines affect the climate indirectly by affecting the distributions of ozone and methane. There is also concern about the impact of contrails and cirrus clouds due to air traffic. Contrails are clouds that are visible trails of water vapor made by the exhaust of aircraft engines. Persistent contrails reduce incoming solar radiation and outgoing thermal radiation in a way that accumulates heat ${ }^{5}$. The emission of water vapor from the aircraft is small relative to all contributions of water vapor to the atmosphere, but may be of concern at higher altitudes.

The modeling of aircraft emissions and their interaction with each other to change the concentration levels of different gasses in the atmosphere and the resulting impact of the radiative forcing on the equilibrium of the Earth's atmosphere is complex and requires the use of coupled atmosphere-ocean general circulation models together with three-dimensional models of carbon cycle and chemistry of other non- $\mathrm{CO}_{2}$ greenhouse gases. These models are computationally intensive and unsuitable for studies involving the generation of multiple scenarios. Simple emission and climate models, based on the input/output relations of linear systems, capture the fundamental emission to climate impact behavior by careful selection of key variables and their dynamics.

The impact of various greenhouse gases depends on the total concentration, effect per unit change in atmospheric concentration and the spatial distribution of the gas. All these quantities are influenced by the lifetime of the gas and depend on the interval of assessment, which may vary from a few decades to a few centuries. Climate metrics ${ }^{6}$ are aimed at providing a common scale to compare different greenhouse gases. If the metrics are to be used as a tool in developing and evaluating aviation operations, they should be transparent and easy to apply. Global Warming Potential (GWP) and Aggregate Global Temperature Potential (AGTP) are some of the commonly used metrics.

This paper integrates a national-level air traffic simulation and optimization capability with simple climate models and carbon cycle models, and climate metrics to assess the impact of aviation on climate. Several authors ${ }^{7-8}$ have used linear climate models and metrics. However, the simulation and analysis capability described in this paper provides the ability to look at the impact of aviation operations on air traffic metrics such as airport arrival and departure rates, sector congestion and air traffic delays while providing the environmental impact metrics using linear models.

Section II provides background for simulating air traffic using flight information and atmospheric and airspace data. Section III describes the aircraft fuel consumption and emission models. Section IV provides the model for diagnosing regions of airspace that are susceptible for persistent contrail formation. Section V explains the optimal trajectory generation with environmental constraints for cruising aircraft. Section VI models the climate response to aviation induced emission and contrails in terms global surface temperature change. Section VII describes metrics to measure the climate impact of aviation emissions and contrails with very different characteristics. Section VIII presents results based on the application of the trajectory optimization algorithm for calculating wind-optimal and contrails-avoidance routes and the climate response model that assesses climate response to air traffic along these routes. Conclusions and future work are described in Section IX.

\section{Air Traffic System Model}

The air traffic system model uses flight information and atmospheric and airspace data as inputs and simulates air traffic in the National Air Space (NAS). Air traffic is modeled using the Future Air Traffic Management Concepts Evaluation Tool (FACET). FACET is a flexible simulation environment for exploration, development, and evaluation of advanced Air Traffic Management (ATM) concepts. FACET models en route airspace operations over the contiguous United States. Core features include air traffic simulation, prediction, visualization, and playback of actual traffic data. Four-dimensional aircraft trajectories are modeled using spherical-earth equations. Aircraft are flown along flight plan or great circle routes as they climb, cruise, and descend according to performance models.

Aircraft in FACET are modeled as parametric 3-dimensional trajectories using an inertial reference system. Values of the parameters vary depending on the aircraft. The equations of motion can be defined by the equations,

$$
\begin{aligned}
& \dot{x}=V \cos \varphi \cos \gamma+u(x, y), \\
& \dot{y}=V \sin \varphi \cos \gamma+v(x, y), \\
& \dot{E}=(T-D) V / m g, \\
& \dot{h}=V \sin \gamma, \\
& \dot{\gamma}=(L \cos \phi-m g \cos \gamma) / m V, \\
& \dot{\varphi}=L \sin \phi / m V \cos \gamma, \\
& \dot{m}=-\sigma(h, V, T),
\end{aligned}
$$


where $x$ is the downrange position, $y$ is the cross track position, $E$ is the energy height, $h$ is the altitude, $\gamma$ is the flight path angle, $\phi$ is the roll angle, $\varphi$ is the heading angle, $m$ is the aircraft mass, Th is the aircraft thrust, $D$ is the drag and $\mathrm{V}$ is the airspeed. The fuel flow $\sigma$ is a function of thrust, altitude and airspeed. $u(x, y)$ and $v(x, y)$ are the $\mathrm{x}$ - and $y$-components of the wind.

\section{Aircraft Fuel Consumption and Emissions Model}

Eurocontrol's Base of Aircraft Data Revision 3.6 (BADA) ${ }^{9}$ provides the fuel consumption model for computing aircraft fuel burn. The following equation calculates fuel burn for aircraft

$$
f=t \cdot S F C \cdot T h
$$

where $f$ is the fuel, $t$ is elapsed time, Th is thrust, and $S F C$ is the specific fuel consumption.

The emission model is based on the Aviation Environmental Design Tool (AEDT) ${ }^{10}$ developed by the Federal Aviation Administration (FAA). Six emissions are computed including $\mathrm{CO}_{2}, \mathrm{H}_{2} \mathrm{O}, \mathrm{SO} x, \mathrm{CO}, \mathrm{HC}$ and $\mathrm{NO} x$. Emissions of $\mathrm{CO}_{2}, \mathrm{H}_{2} \mathrm{O}$ and $\mathrm{SO} x$ (modeled as $\mathrm{SO}_{2}$ ) are directly proportional to fuel consumption ${ }^{11}$. The emissions are computed by

$$
\begin{aligned}
& E_{\mathrm{CO}_{2}}=E I_{\mathrm{CO}_{2}} \cdot f=3155 \cdot f, \\
& E_{\mathrm{H}_{2} \mathrm{O}}=E I_{\mathrm{H}_{2} \mathrm{O}} \cdot f=1237 \cdot f, \\
& E_{\mathrm{SO}_{2}}=E_{\mathrm{SO}_{2}} \cdot f=0.8 \cdot f .
\end{aligned}
$$

The terms $E_{\mathrm{CO}_{2}}, \mathrm{EI}_{\mathrm{CO}_{2}}, E_{\mathrm{H}_{2} \mathrm{O}}, E I_{\mathrm{H}_{2} \mathrm{O}}$ and $E_{\mathrm{SO}_{2}}, E_{\mathrm{SO}_{2}}$ are emissions and emission index of $\mathrm{CO}_{2}, \mathrm{H}_{2} \mathrm{O}$ and $\mathrm{SO}_{2}$. The emissions are in grams, and fuel burn is in kilograms. Emissions of $\mathrm{CO}, \mathrm{HC}$ and NOx are modeled through the use of the Boeing Fuel Flow Method 2 (BFFM2) ${ }^{12}$. The emissions are determined by aircraft engine type, altitude, speed, and fuel burn and the coefficients in the International Civil Aviation Organization (ICAO) emission data bank. The climate response model in the next section assesses the impact of aircraft emissions on climate change.

\section{Persistent Contrails Formation Models}

The formation of contrails has been under investigation since $1919 .{ }^{13}$ According to Appleman ${ }^{14}$, contrails are clouds of tiny ice particles that form when a mixture of warm engine exhaust gases and cold ambient air reaches saturation with respect to water. Contrails form in the regions of airspace that have ambient Relative Humidity with respect to Water (RHw) greater than a critical value, $r_{\text {contr }}{ }^{15}$. Contrails can persist when the ambient air is supersaturated with respect to ice i.e. the environmental Relative Humidity with respect to Ice (RHi) is greater than $100 \%{ }^{2}$ In this study, the regions of airspace that have RHw greater than $r_{\text {contr }}$ and RHi greater than $100 \%$ are considered favorable to persistent contrails formation. More details for the contrails prediction model and the weather forecasting system, Rapid Update Cycle (RUC), are provided in a previous paper ${ }^{16}$.

This model will be used to compute the regions in the U. S. airspace that facilitate persistent contrails formation. The blue, green and magenta polygons in Fig. 1 depict the areas at 37,000 feet above sea level in the U.S. national airspace where atmospheric conditions are favorable for persistent contrails formation at 6 a.m., 7 a.m. and 8 a.m. EDT on May 24, 2007, respectively. The critical relative humidity and RHi values are computed using the RHw values, pressure, and temperature data obtained from RUC. Figure 1 shows that the location, shape and size of potential contrail regions vary with time.

\section{Aircraft Trajectories with Environmental Constraints}

Wind-optimal trajectories are fuel-efficient trajectories between any two city-pairs. They are also environmentally friendly since most emissions are directly proportional to fuel usage. However, if the trajectory goes through a region where the atmospheric conditions are favorable to persistent contrail formation, contrails create additional impact on the climate. The amount of contrail formation can be reduced either by flying around the potential contrail regions at the same cruise altitude ( $2 \mathrm{D}$ approach) or by changing the cruise altitude and going 
around the potential contrail regions (3D approach). The amount of additional fuel consumed in flying a contrail reduction route instead of flying a wind optimal route represents increased cost to the airlines as well as a negative impact on the environment due to the resulting excess $\mathrm{CO}_{2}$ emissions. Each additional $\mathrm{kg}$ of fuel produces approximately $3.15 \mathrm{~kg}$ of $\mathrm{CO}_{2}$. The optimization module in the simulation generates trajectories that reduce travel time through contrail regions by imposing a penalty on travel through potential contrail areas ${ }^{16}$.

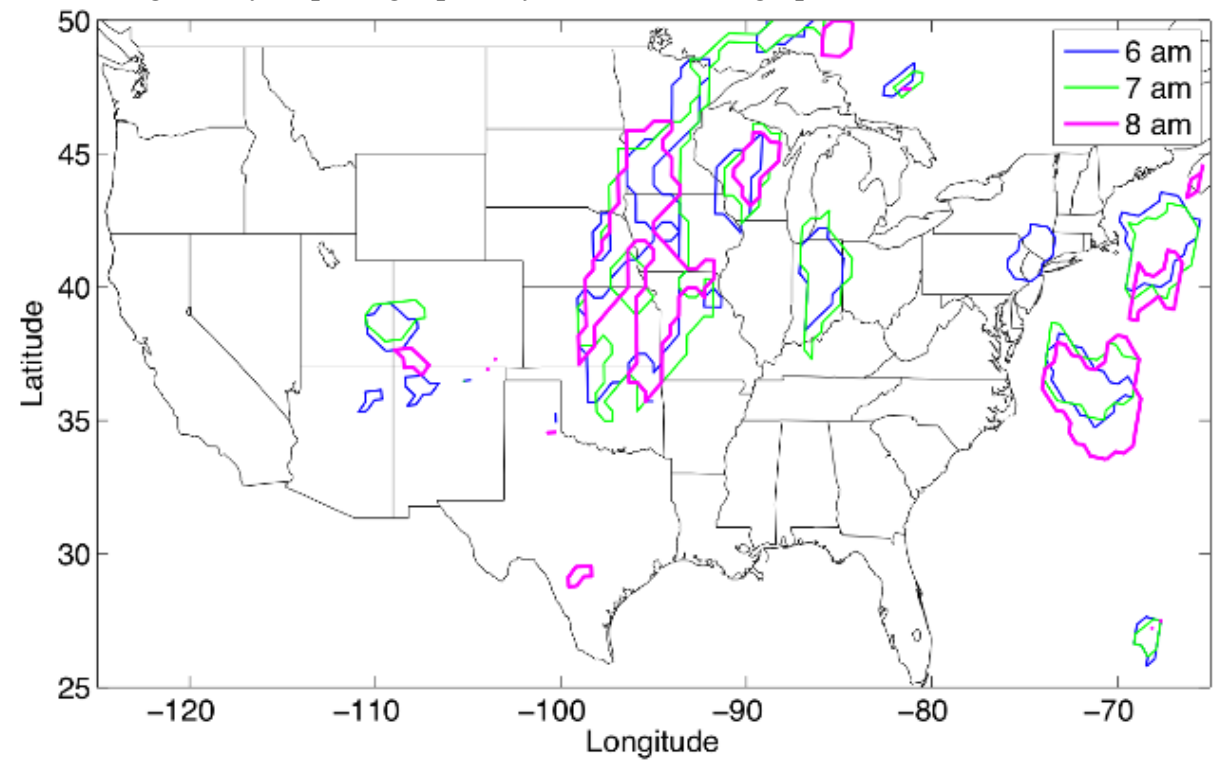

Figure 1. Regions of airspace at 37,000 feet favorable for persistent contrails formation between 6 a.m. and 8 a.m. on May 24, 2007.

Three optimal trajectories from Chicago O'Hare airport (ORD) to Newark Liberty airport (EWR), corresponding to three different penalties on the contrail regions, are shown in Fig. 2 for flights with cruising altitude equal to 34,000 feet. The cruising speed is assumed to be $400 \mathrm{nmi} / \mathrm{hr}(741 \mathrm{~km} / \mathrm{hr})$. The green arrows represent the wind directions, obtained from RUC, at 6 a.m. EDT on May 24, 2007. The arrow sizes are plotted in proportion to the wind magnitudes. The areas favorable to persistent contrails formation (RHi $>100 \%$ ) are indicated by the blue polygons. $C_{r}$ is a weighting factor used to impose a penalty on a trajectory going through a potential contrail region. The wind-optimal trajectory is generated imposing no penalty, $C_{r}=0$, for travel through contrail regions. Two optimal trajectories in additional to the wind-optimal route are also plotted in Fig. 2. The optimal route with $C_{r}=0.6$ completely avoids the contrail polygons near the departure airport. The optimal route with $C_{r}=0.2$ only partially avoids the polygons but is shorter. Note that both routes travel through the blue polygon surrounding the destination where aircraft start to land. In this case, there is a tradeoff between flying a shorter route with more persistent

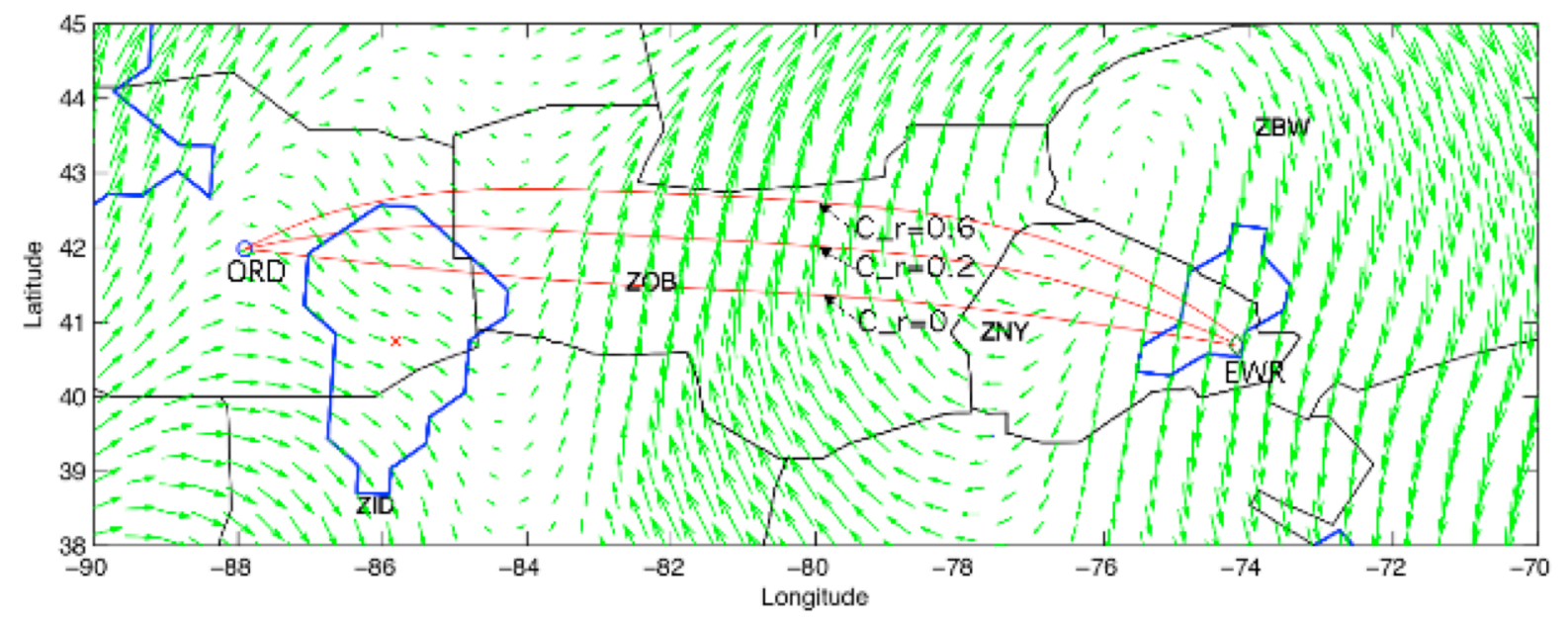

Figure 2. Optimal trajectories at 34,000 feet from ORD to EWR with different penalties on contrail regions. 
contrails formation versus flying a longer route with less persistent contrails formation. The performance of optimal trajectories is evaluated by investigating the total travel time and the time spent traveling through regions of persistent contrails formation.

\section{Linear Emission and Climate Models}

This section models the climate response to aviation emission and contrails as outputs from a series of linear dynamic systems. The linear systems are generated against a background of concentration of various greenhouse gases resulting from past emissions from all sources. Linear emission models provide the incremental changes to the greenhouse gas concentrations resulting from the emission due to some or all aircraft operations.

Several linear models have been proposed for representing the change in the concentration of $\mathrm{CO}_{2}$ due to the emission of a unit amount of $\mathrm{CO}_{2}$. The carbon cycle models describe the changes to the $\mathrm{CO}_{2}$ concentration due to the transport and absorption of $\mathrm{CO}_{2}$ by the land mass and various ocean layers. A $19^{\text {th }}$ order model with states representing the interaction between different oceans and depths is described in the literature ${ }^{17}$. This paper uses a $3^{\text {rd }}$ order linear system to describe the $\mathrm{CO}_{2}$ concentration dynamics and the model parameters will be described in subsequent sections. The concentration dynamics of other non- $\mathrm{CO}_{2}$ greenhouse gases are described by first order linear systems. Radiative forcing (RF) associated with $\mathrm{CO}_{2}$ and other gases can be adequately approximated by simple functions of concentrations ${ }^{18-19}$.

Radiative Forcing due to different emissions affects the climate by changing the global average temperature. The temperature response/energy balance to RF can be modeled using either a first order linear model ${ }^{20}$ or a second order linear model ${ }^{21}$. The time constants in the two-box ocean model correspond to the dynamics associated with the surface layers of the ocean and the thermal inertia associated with the deep ocean. A comparison of different linear models used to estimate the impact of aviation on climate suggests that multi-box ocean model gives closer approximation to ocean temperature response ${ }^{7}$. The modeling of emission to temperature change is illustrated in Figure 3.

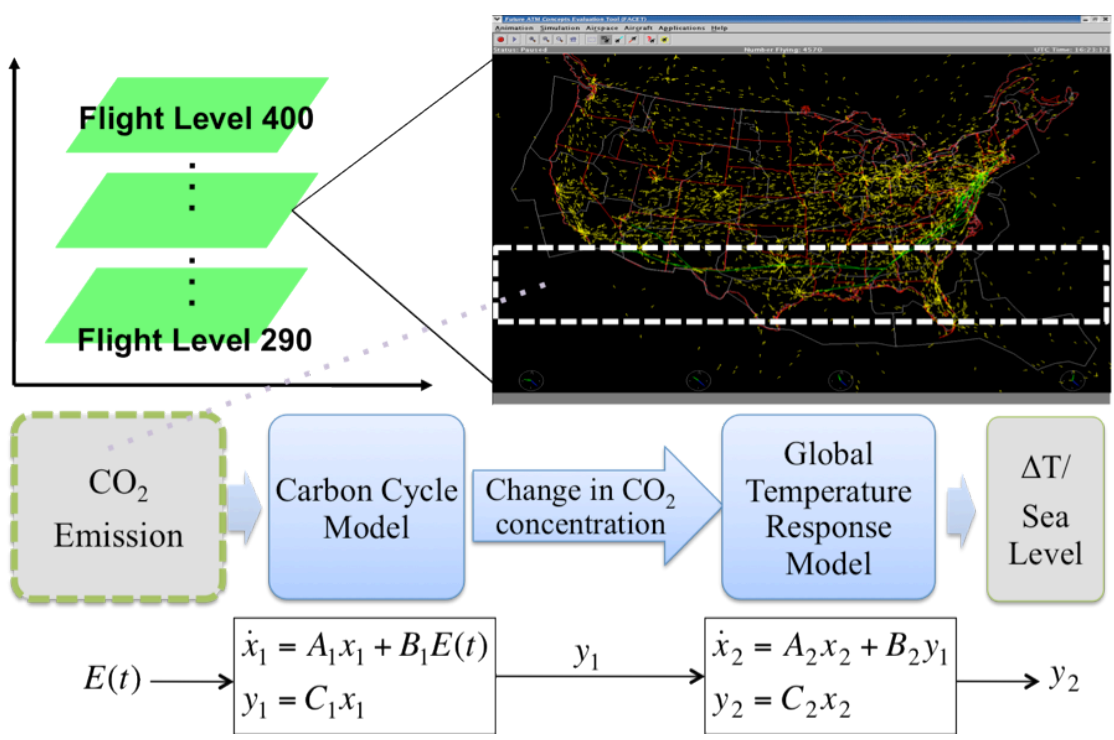

Figure 3. Linear climate response models for aviation induced climate change.

The response to a first order linear system $\frac{d x(t)}{d t}=a x(t)+b u(t)$ where $\mathrm{x}(\mathrm{t})$ is the state variable and $\mathrm{u}(\mathrm{t})$ is the forcing function with $\mathrm{a}$ and $\mathrm{b}$ constants is given by the expression

$$
x(t)=e^{a\left(t-t_{0}\right)} x\left(t_{0}\right)+\int_{t_{0}}^{t} e^{a(t-\xi)} b u(\zeta) d \xi
$$


Let $\Delta x(t)$ be the concentration change for a gas in the atmosphere at time $t$ due to a disturbance $\mathrm{u}\left(\mathrm{t}_{0}\right)$ applied at time, $\mathrm{t}_{0}$, resulting from a source of aviation emission. Since it is desirable to estimate the change in concentration, the initial value of $x(t)$ at $t=t_{0}$ can be assumed to be zero. This reduces the solution to

$$
\Delta x(t)=\int_{t_{0}}^{t} e^{a(t-\xi)} b u(\xi) d \xi
$$

The concentration change for a gas due to a unit impulse of gas emitted at $t_{0}$ is obtained by solving Eq. (5) and is equal to

$$
\Delta x(t)=b e^{a\left(t-t_{0}\right)} .
$$

Similarly, the concentration change for a constant unit emission from $\mathrm{t}_{0}$ to $\mathrm{t}$ is $\Delta x(t)=-\frac{b}{a}\left[1-e^{a\left(t-t_{0}\right)}\right]$.

The radiative forcing imposed by the emission can be assumed for the simplest model to be linearly proportional to the concentration change. The change in the radiative forcing is represented by

$\Delta F(t)=\int_{t_{0}}^{t} k \delta(t-\zeta) \Delta x(\zeta) d \zeta=k \Delta x(t)$ that drives a surface temperature change $\Delta T(t)$.

A first-order climate model ${ }^{20}$ proposed in the literature models the temperature change, $\Delta T(t)$, as the following

$$
C \frac{d \Delta T(t)}{d t}=-\frac{\Delta T(t)}{\lambda}+\Delta F(t)
$$

Substituting $a=-1 / \lambda C$ and $b=1 / C$ in Eq. (7), the change in the surface temperature at time $t$ is given by

$$
\Delta T\left(t, t_{0}\right)=1 / C \int_{t_{0}}^{t} e^{-(t-\zeta) / \lambda C} \Delta F(\zeta) d \zeta
$$

The change in the surface temperature to an exponentially decaying radiative forcing of strength $A$, which is in the same form as shown in Eq. (8) and defined as $\Delta F(t)=A e^{-\left(t-t_{0}\right) / \alpha}$, resulting from the emission applied impulsively at the initial time $t_{0}$ is

$$
\Delta T\left(t, t_{0}\right)=1 / C \int_{t_{0}}^{t} e^{-(t-\zeta) / \lambda C} A e^{-\left(\zeta-t_{0}\right) / \alpha} d \zeta
$$

Evaluating the integral, Eq. (9) reduces to

$$
\Delta T\left(t, t_{0}\right)=A \alpha \tau\left[e^{-\left(t-t_{0}\right) / \alpha}-e^{-\left(t-t_{0}\right) / \tau}\right] / C(\alpha-\tau)
$$

where the time scale of climate response $\tau=\lambda C$. C is the heat capacity of the climate system and $\lambda$ is the climate sensitivity parameter. Climate response to aviation emission can be assessed through quantifying global surface temperature change modeled by cascading several first order systems. The following subsections introduce a recently developed climate assessment metric based on linear climate systems for modeling temperature change with higher order systems.

\section{Climate Metrics}

Aviation emissions and contrails have very different characteristics and influence the climate either directly or by decomposition into other chemical compounds. Figure 4 shows the lifetime associated to contrails, ozone, and 
$\mathrm{CO}_{2}$ emission. The impact of certain gases depends both on the amount of emission and the location of the emission. In addition, the impact of the emissions varies with the choice of the time horizon. These variations make it necessary to develop a common yardstick to measure the impact of various gases. Several climate metrics have been developed to assess the impact of aviation emissions. These metrics have been used to develop strategies for limiting the impact on the environment ${ }^{6,22}$.

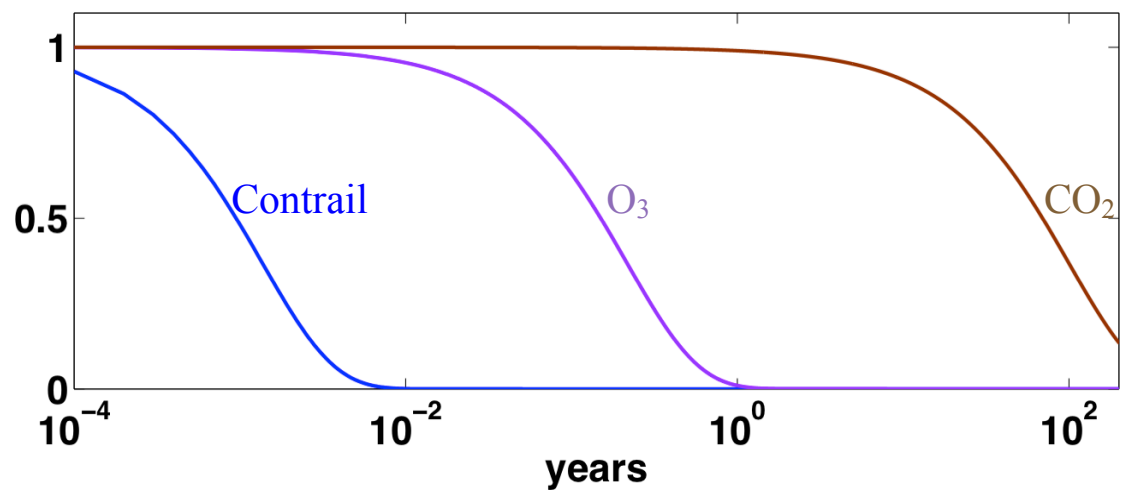

Figure 4. Lifetime associated to contrails, ozone, and $\mathrm{CO}_{2}$ emission.

\section{A. Global Temperature Change Potential}

Global Temperature Change Potential (GTP) $)^{23}$ is a climate assessment metric that adapts a linear system for modeling the global temperature response to aviation emissions and contrails. The definition of the absolute GTP is a convolution integral from $t_{0}=0$ to $t=H$, and has the following representation,

$$
A G T P(H)=\int_{0}^{H} R(H-\zeta) \Delta F(\zeta) d \zeta
$$

where $R(H-\zeta)$ is the impulse response function for the surface temperature change at time $H$ due to a radiative forcing applied at $\zeta$. Note that surface temperature change $\Delta T\left(t, t_{0}\right)$ by the simplified climate model in Eq. (7-10) is equivalent to the $\operatorname{AGTP}(H)$ when setting $t=H, t_{0}=0, R(H-\zeta)=\frac{1}{C} e^{-(H-\zeta) / \lambda C}$, and $\Delta F(\zeta)=A e^{-\zeta / \alpha}$. Two versions of AGTP are available in the literature. The pulse AGTP measures the change in the global temperature at a particular time, $t$, in the future due to an instantaneous disruption at $t_{0}$. The sustained AGTP measures the global temperature change at time $t$ due to disruptions constantly applied for a period between $t$ and $t_{0}$.

AGTP is employed in this study for translating aviation induced $\mathrm{CO}_{2}$ emission and persistent contrails into total effect on global warming. In general, higher order systems are adopted for modeling the impulse response function $R(H-\zeta)$ and the radiative forcing $\Delta F(\zeta)$. The formulations for AGTP due to $\mathrm{CO}_{2}$ emission and contrails are provided in the following subsections.

\section{B. Pulse and Sustained AGTP for $\mathrm{CO}_{2}$ emission} by

The response of global-mean surface temperature to a unit impulse of $\Delta F$, using a second order model ${ }^{21}$ is given

$$
R(t)=\sum_{j=1}^{2} \frac{c_{j}}{d_{j}} e^{-t / d_{j}}
$$

where the parameters $c_{j}$ and $d_{j}$ are given in Table 1 taken from the literature ${ }^{6}$. The first term and second term in the summation are associated with the response of the ocean mixed-layer and Deep Ocean, respectively, to a forcing. The radiative forcing for $\mathrm{CO}_{2}$ emission is made of a steady state component and three exponentially decaying components, and is given by 


$$
\Delta F^{C O_{2}}(t)=A^{C O_{2}}\left(a_{0}+\sum_{i=1}^{3} a_{i} e^{-\left(t-t_{0}\right) / \alpha_{i}}\right)
$$

where $A^{\mathrm{CO}_{2}}=1.82 \times 10^{-15} \mathrm{Wm}^{-2} \mathrm{~kg}^{-1}$ is the specific forcing due to $\mathrm{CO}_{2}$ and its value is taken from past studies ${ }^{24}$. The exponential component in parentheses is the decay of an impulse emission of $\mathrm{CO}_{2}$ with time and the parameters are shown in Table 1.The expression for AGTP given in Eq. (11) is evaluated after substituting Eq. (12-13).

The pulse AGTP for $1 \mathrm{~kg} \mathrm{CO}_{2}$ emission for a time horizon $H$ can then be found by analytical integration using Eq. (11-13), and is given by

$$
A G T P^{C O_{2}}(H)=A^{C O_{2}} \sum_{j=1}^{2}\left[a_{0} c_{j}\left(1-e^{-H / d_{j}}\right)+\sum_{i=1}^{3} \frac{a_{i} \alpha_{i} c_{j}}{\alpha_{i}-d_{j}}\left(e^{-H / \alpha_{i}}-e^{-H / d_{j}}\right)\right] .
$$

This measures the surface temperature change due to $\mathrm{CO}_{2}$ emitted at a particular time instant at $t=0$.

The sustained AGTP for $\mathrm{CO}_{2}$ models the temperature change due to $\mathrm{CO}_{2}$ emitted constantly over a period of time between $t=0$ and $t=H$. For the same amount of $\mathrm{CO}_{2}$ emissions, the sustained AGTP is formulated as

$$
\begin{aligned}
& \frac{1}{H} \int_{0}^{H} A G T P^{C O_{2}}(\tau) d \tau \\
& \quad=\frac{A^{C O_{2}}}{H} \sum_{j=1}^{2}\left\{a_{0} c_{j}\left[H-d_{j}\left(1-e^{-H / d_{j}}\right)\right]+\sum_{i=1}^{3} \frac{a_{i} \alpha_{i} c_{j}}{\alpha_{i}-d_{j}}\left[\alpha_{i}\left(1-e^{-H / \alpha_{i}}\right)-d_{j}\left(1-e^{-H / d_{j}}\right)\right]\right\} .
\end{aligned}
$$

Table 2 shows the pulse and sustained AGTP values per kilogram of $\mathrm{CO}_{2}$ emission for three time horizons. The absolute global temperature potentials are expressed in terms of the specific forcing, $A^{\mathrm{CO}_{2}}$, since different values can be selected from the literature.

\section{Pulse and Sustained AGTP for Persistent Contrails}

The surface temperature response for contrails is modeled similarly. An impulse function best characterizes contrails radiative forcing since contrails are short-lived, usually last for several hours, in the atmosphere. The pulse AGTP for contrails formation is simply taken as the impulse response. For a unit of contrails induced radiative forcing, the pulse AGTP is represented by

$$
\operatorname{AGTP}^{\text {Contrails }}(H)=\int_{0}^{H} R(H-\zeta) \delta(\zeta-0) d \zeta=R(H) .
$$

The sustained version for AGTP ${ }^{\text {Contrails }}$ is obtained by integrating $R(t)$ over the period of $H$,

$$
\frac{1}{H} \int_{0}^{H} R(\zeta) d \tau=\frac{1}{H} \sum_{j=1}^{2}\left[c_{j}\left(1-e^{-H / d_{j}}\right)\right]
$$

Equation (16-17) computes surface temperature change to a unit of energy induced to the atmosphere by contrails. The net radiative forcing for contrails includes the longwave $R F_{L W}$ and the shortwave $R F_{S W}$ radiative forcing and is defined as $R F_{\text {nets }}=R F_{L W}+R F_{S W}$. It is measured in terms of unit of power (Watts) per unit area of contrails $\left(\mathrm{m}^{2}\right)$. Typical values for $R F_{\text {nets }}$ have a range between $10 \mathrm{Wm}^{-2}$ and $30 \mathrm{Wm}^{-2}$ taken from Meerkötter ${ }^{5}$ and Haywood $^{25}$. Due to the nature of contrail formation, it is argued that it is better to represent contrail radiative forcing in terms of unit distance flown by the aircraft (watts $/ \mathrm{km}$ ). The amount of energy, $E F$, induced to the atmosphere for a unit length of contrail over its lifetime is defined as ${ }^{26}$ 


$$
E F=\int_{\text {lifetime }} R F_{\text {nets }}(\xi) W_{c}(\xi) d \xi
$$

where $W_{c}$ is contrail width (m). Suppose $R F_{\text {nets }}=10 \mathrm{Wm}^{-2}$, contrail width $W=1000 \mathrm{~m}$ and contrails lifetime is 10000 s, the energy $E F$ for a $\mathrm{km}$ contrail equals 100 Gigajoules (GJ). The global surface temperature change, $\operatorname{AGTP}^{\text {Contrails }}(H)$, is then computed by multiplying either Eq. (16) or Eq. (17) by the energy after it is normalized by the surface area of the Earth and total seconds in a year. Assuming $E F=100 \mathrm{GJ}$ per kilometer of contrails, the pulse and sustained AGTP values are computed for 3 time horizons using equations 12,16 and 17. The AGTP values are shown in Table 2 . Note that they can be linearly scaled when other $R F_{\text {nets }}$ values are chosen.

Table 1. Parameter values for the radiative forcing function due to $\mathrm{CO}_{2}$ emission and impulse response functions in AGTP.

\begin{tabular}{|c|c|c|c|c|}
\hline Index & 0 & 1 & 2 & 3 \\
\hline$a_{i}$ (unitless) & 0.217 & 0.259 & 0.338 & 0.186 \\
\hline$\alpha_{i}($ Years $)$ & & 172.9 & 18.51 & 1.186 \\
\hline$c_{j}\left(\mathrm{~K} / \mathrm{Wm}^{-2}\right)$ & & 0.631 & 0.429 & \\
\hline$d_{j}$ (years) & & 8.4 & 409.5 & \\
\hline
\end{tabular}

Table 2. Absolute global temperature potential values for $\mathrm{CO}_{2}$ emission and contrails.

\begin{tabular}{|c|c|c|c|}
\hline Horizon (years) & $\mathrm{H}=25$ & $\mathrm{H}=50$ & $\mathrm{H}=100$ \\
\hline $\begin{array}{c}\text { Pulse } A G T P^{\mathrm{CO}_{2}}(H) \\
(\mathrm{K} / \mathrm{kg})\end{array}$ & $A^{\mathrm{CO}_{2}} \times 0.3686$ & $A^{\mathrm{CO}_{2}} \times 0.3176$ & $A^{\mathrm{CO}_{2}} \times 0.2788$ \\
\hline $\begin{array}{c}\text { Sustained } A G T P^{\mathrm{CO}_{2}}(H) \\
(\mathrm{K} / \mathrm{kg})\end{array}$ & $A^{\mathrm{CO}_{2}} \times 0.3024$ & $A^{\mathrm{CO}_{2}} \times 0.3223$ & $A^{\mathrm{CO}_{2}} \times 0.3079$ \\
\hline $\begin{array}{c}\text { Pulse } A G T P^{\text {Contrails }}(H) \\
(\mathrm{K} / \mathrm{km})\end{array}$ & $2.9936 \mathrm{e}-14$ & $6.9778 \mathrm{e}-15$ & $5.1045 \mathrm{e}-15$ \\
\hline $\begin{array}{c}\text { Sustained } A G T P^{\text {Contrails }}(H) \\
(\mathrm{K} / \mathrm{km})\end{array}$ & $1.5522 \mathrm{e}-13$ & $8.4377 \mathrm{e}-14$ & $4.5003 \mathrm{e}-14$ \\
\hline
\end{tabular}

$\mathrm{CO}_{2}$ is the most pervasive of all anthropogenic emissions. The impact of the introduction of additional amount of $\mathrm{CO}_{2}$ on climate is better understood than the impact of all other greenhouse gases and contrails. In the development of climate metrics, the impact of other emissions is normalized with respect to the impact of $\mathrm{CO}_{2}$. The atmosphere quickly absorbs additional $\mathrm{CO}_{2}$ and the $\mathrm{CO}_{2}$ radiative forcing is due to the globally distributed energy resulting from the uniform increase in $\mathrm{CO}_{2}$ concentration. Contrails occur at different regions of the earth and add non-uniform sources of energy to the atmosphere. The efficacy factor is used to differentiate the way radiative forcing from $\mathrm{CO}_{2}$ and contrails affect the climate. Specifically, it is defined as the ratio between the global temperature increase for a local energy input relative to that for a $\mathrm{CO}_{2}$-equivalent globally distributed energy input ${ }^{26}$. The efficacy factor for annual mean contrail cover is estimated to be about $0.6^{27}$ and treated as a parameter in this paper ranging between 0.6 and1.0.

\section{Results}

This section presents results based on using the simulation and analysis capability described in the previous sections. The optimal trajectory algorithm is applied to calculate an aircraft trajectory in the presence of winds that minimizes fuel burn and avoids regions of airspace that facilitate persistent contrails formation. The potential impacts to the climate for the U.S. domestic flights between 12 origin-destination pairs are assessed in terms of mean surface temperature change, i.e., AGTP due to aircraft emissions and persistent contrails formation. The same city-pairs were used by the Federal Aviation Administration to assess the impact of implementation of Reduced Vertical Separation Minima (RVSM) on aircraft-related fuel burn and emissions ${ }^{28}$. This study adapts the standard in RVSM and assumes that the cruising altitudes are between 29,000 and 41,000 feet. Eastbound aircraft fly odd thousands of feet while westbound traffic fly even thousands of feet. Figure 5 shows the wind-optimal trajectories for the eastbound flights at 37,000 feet at 6 a.m. Eastern Daylight Time (EDT) on May 24, 2007. Blue polygons 
depict the areas favorable to persistent contrails formation. The trajectory computations are done using traffic and atmospheric data in the continental United States for May 4, May 24, and May 27 in 2007. The data for wind speed and direction are obtained from RUC.

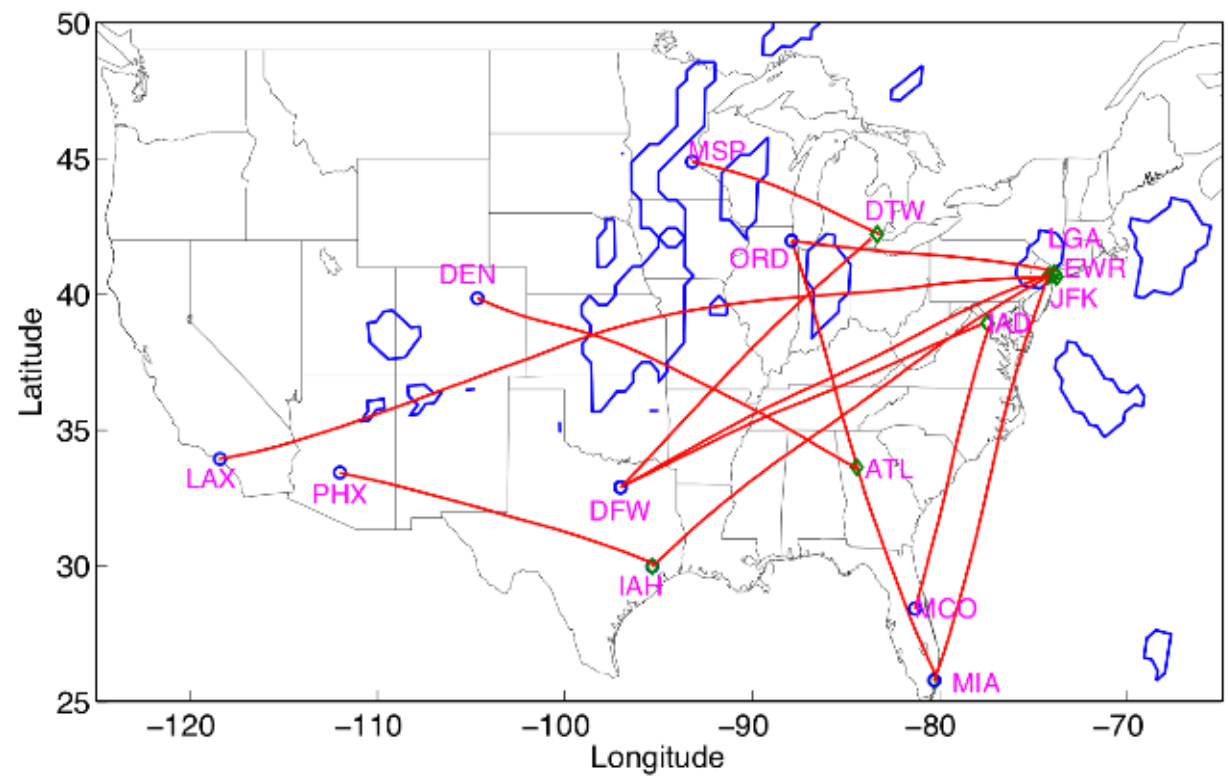

Figure 5. The wind-optimal trajectories for the eastbound flights for 12 city pairs at 37,000 feet, 6 a.m. EDT on May 24, 2007.

\section{Persistent Contrails Formation in May, 2007}

Great-circle trajectories between the 12 city pairs are simulated to assess the severity of potential contrail formation induced by the air traffic throughout May in 2007. The results, shown in Fig.6, is used to identify the days in May, 2007 that have low, medium and high potential contrail formation for the 12 city pairs. Then, climate impact of air traffic is investigated in more detail for these days. Persistent contrail-favorable regions over the continental United States are computed ${ }^{16}$ for May 2007 using atmospheric data from the RUC. A flight along the great-circle route at the assigned flight-plan altitude between the 12 city pairs is simulated for each hour thorough May 2007 since atmospheric data are updated hourly. The total time that aircraft fly inside contrail-favorable regions is recorded each day. Figure 6 shows the average contrail formation time for a group of 24 flights traveling between the 12 city pairs. May 24 has low (55 minutes) contrail formation, May 4 has medium contrails formation, and May 27 has the highest (493 minutes) contrail formation.

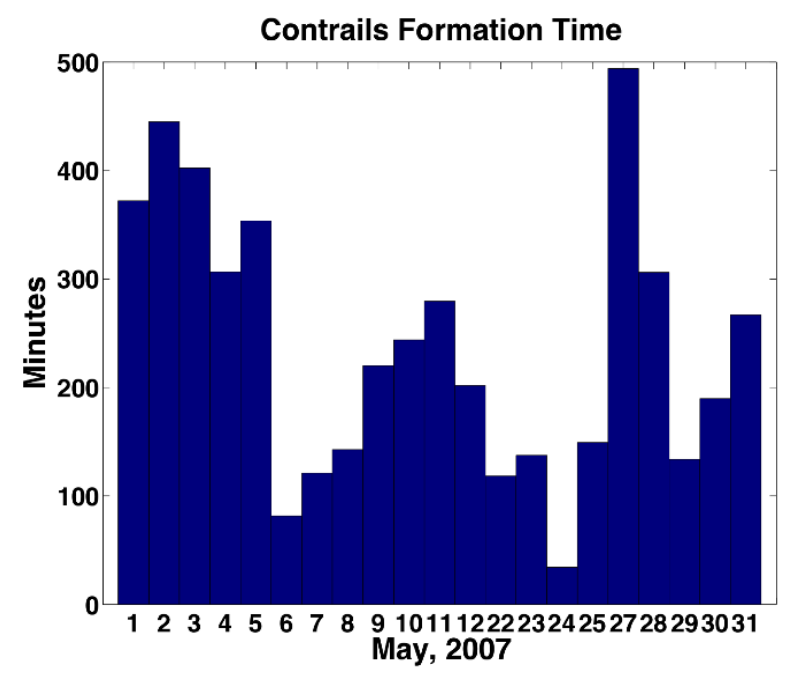

Figure 6. Average persistent contrail formation time for each day in May 2007.

10

American Institute of Aeronautics and Astronautics 


\section{Trade-off between AGTP and Additional Fuel Burn}

This section analyzes climate impact of the wind-optimal and contrails-avoidance trajectories for 12 origindestination pairs on low, medium, and high contrails formation day. The optimal aircraft trajectories are generated at the beginning of each hour for a period of 24 hours starting from 6 a.m. EDT on May 24, 4, and 27 in 2007, respectively, using hourly updated weather data from RUC. Six flight levels are considered for each direction of air traffic for each city pair. A group of 21 optimal aircraft trajectories are calculated for each flight level by increasing the value of $C_{r}$ from 0 to 2 with increments equal to 0.1 . The cost coefficient of time is chosen as $C_{t}=20$ for each case. The cruising speed is assumed to be $420 \mathrm{nmi} / \mathrm{hr}$. The fuel consumption for each aircraft trajectory is calculated using Base of Aircraft Data ${ }^{9}$ (BADA) formulas by assuming that the aircraft are short to medium range jet airliners with medium weight. $\mathrm{CO}_{2}$ emission is then obtained from fuel burn using the emission index. The persistent contrails formation time associated with each trajectory is also recorded. The details of aircraft trajectory generation are described in an earlier paper ${ }^{16}$.

A total of fifty bins are defined such that the aircraft trajectories can be categorized based on their additional fuel consumption. The first bin contains the wind-optimal trajectory, which is the baseline for fuel use comparison and corresponds to trajectories that require zero \% of additional fuel consumption. The second bin contains aircraft trajectories that consume less than $1 \%$ additional fuel, the third bin contains those consuming less than $2 \%$, and etc. The fiftieth bin has trajectories that burn more than $49 \%$ of fuel. In each bin, the optimal trajectory that has least amount of persistent contrails formation time is selected to represent the bin. Note that there are fifty bins for each group of trajectories and six groups for each direction of air traffic every hour.

The surface temperature change due to $\mathrm{CO}_{2}$ emission is calculated for the trajectories in each bin. Figure 7 shows the AGTP values and fuel burn for flights between the 12 city pairs on May 27, 2007. The average fuel burn for each set of 24 flights along the wind-optimal trajectories is about 130 tons that is converted to three AGTP values for time horizons of 25 years, 50 years, and 100 years. The climate response (AGTP) to $\mathrm{CO}_{2}$ emission diminishes as the time horizon increases and increases linearly with the fuel burn for each horizon.

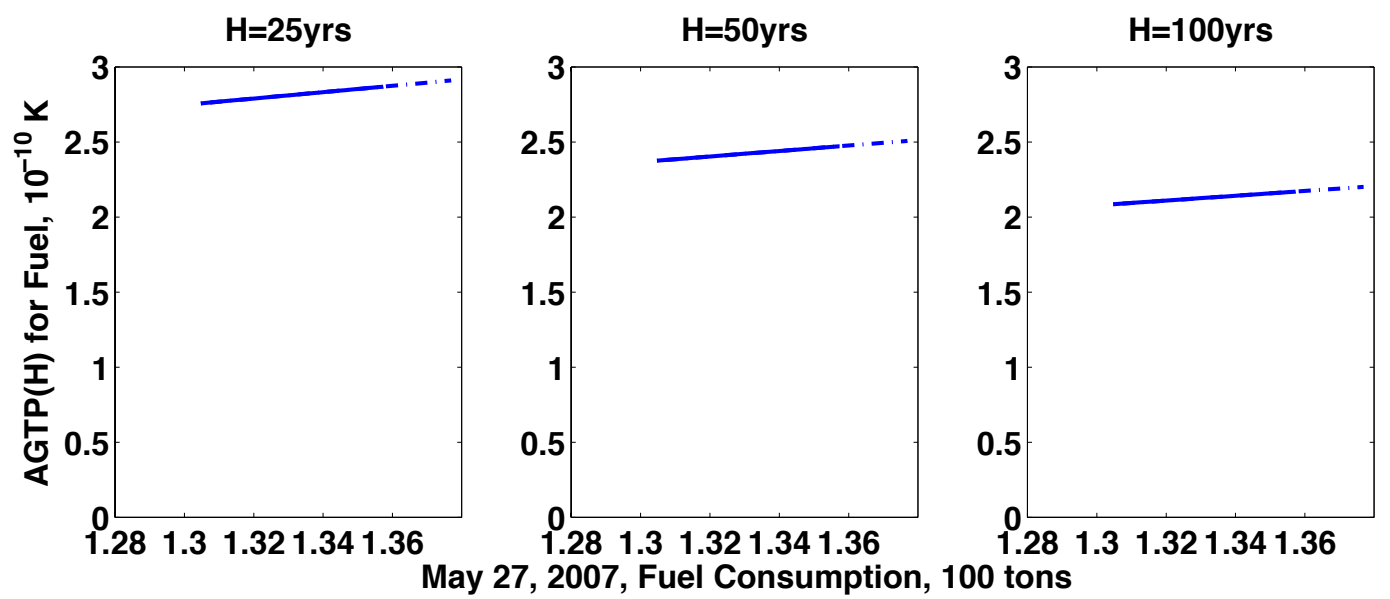

Figure 7. Surface temperature change due to fuel burn for May 27, 2007.

There is significant uncertainties ${ }^{29-31}$ with the radiative forcing associated with contrails and the equivalent energy forcing. The surface temperature changes due to contrails formation associated with three different radiative forcing values, which are $30 \mathrm{Wm}^{-2}, 10 \mathrm{Wm}^{-2}$, and $3.3 \mathrm{Wm}^{-2}$ are plotted in magenta, blue, and green in Figure 8 . The dash-dot and solid curve show results for flights with and without altitude optimization, respectively. The error bars indicate the variation of AGTP due to contrails as the efficacy factor is varied from 1 to 0.6 . The figure shows when altitude is optimized, about 6 tons increase in total fuel consumption can reduce the surface temperature change due to contrails to $0 \mathrm{~K}$ from $5.7 \times 10^{-10} \mathrm{~K}, 1.9 \times 10^{-10} \mathrm{~K}, 0.63 \times 10^{-10} \mathrm{~K}$ for various radiative forcing values when time horizon is 25 years. Without altitude optimization, the reduction in AGTP values due to contrails is more gradual with increase in total fuel consumption. Allowing further increase in fuel consumption does not result in proportionate reduction in contrail induced AGTP. The AGTP values are smaller but the trends remain the same for these curves when a longer time horizon is considered. 

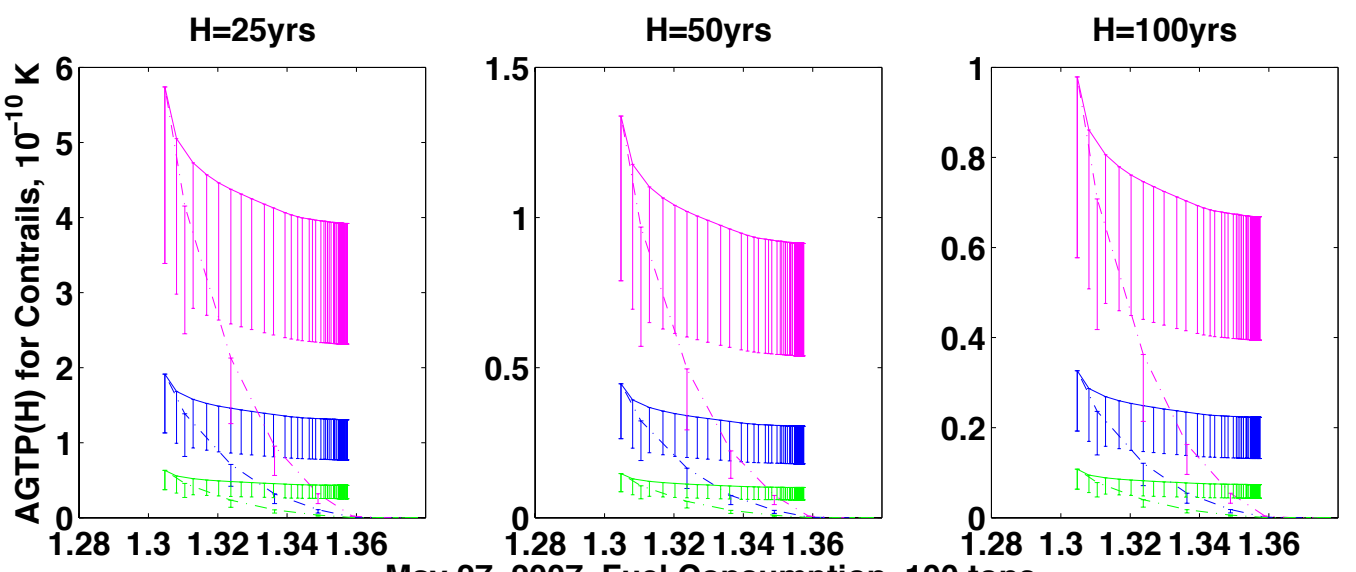

Figure 8. Surface temperature change due to contrails formation for May 27, 2007.

Figure 9 shows the total surface temperature response to both the $\mathrm{CO}_{2}$ emissions and contrails. Without optimizing the altitudes, the set of trajectories that burns 136 tons of fuel acquire the minimum total surface temperature change, $6.79 \times 10^{-10} \mathrm{~K}$, for 25 years time horizon when contrails radiative forcing value is $30 \mathrm{Wm}^{-2}$. The sets of trajectories that burn 135 tons and 133 tons have the smallest AGTP values, $4.17 \times 10^{-10} \mathrm{~K}$ and $3.28 \times 10^{-10} \mathrm{~K}$, when the radiative forcing are $10 \mathrm{Wm}^{-2}$ and $3.3 \mathrm{Wm}^{-2}$. Burning extra $4.6 \%, 3.9 \%$, and $2.3 \%$ fuel reduce the AGTPs by $20 \%, 11 \%$, and $3.2 \%$, for the 3 radiative forcing values for 25 years time horizon. Aircraft trajectories selected for minimum temperature change are closer to wind-optimal when contrails radiative forcing is smaller. Because wind-optimal trajectories minimize $\mathrm{CO}_{2}$ emission that has a life time much longer than contrails, similar trends are observed when a longer time horizon is considered. The minimum AGTP values are $2.89 \times 10^{-10} \mathrm{~K}, 2.88 \times 10^{-10} \mathrm{~K}$, and $2.87 \times 10^{-10} \mathrm{~K}$ for the three contrails radiative forcing when attitude is optimized. The associated fuel burn values are about 137 tons, 136 tons and 136 tons. In this case, burning extra 5.4\%, 4.6\%, and 4.6\% fuel reduce the AGTPs by $66 \%, 38 \%$, and $15 \%$, respectively. When 100 years time horizon is considered, $4.6 \%, 4.6 \%$, and $3.1 \%$ extra fuel burn reduces the AGTPs by $29 \%, 10 \%$, and $1.8 \%$, respectively. The temperature changes are almost entirely due to $\mathrm{CO}_{2}$ emission since these trajectories almost completely avoid contrail favorable areas. Aircraft, with optimized altitude, are also flying closer to wind-optimal for minimum temperature change when contrails radiative forcing is smaller or horizon is longer for the same aforementioned reasons.

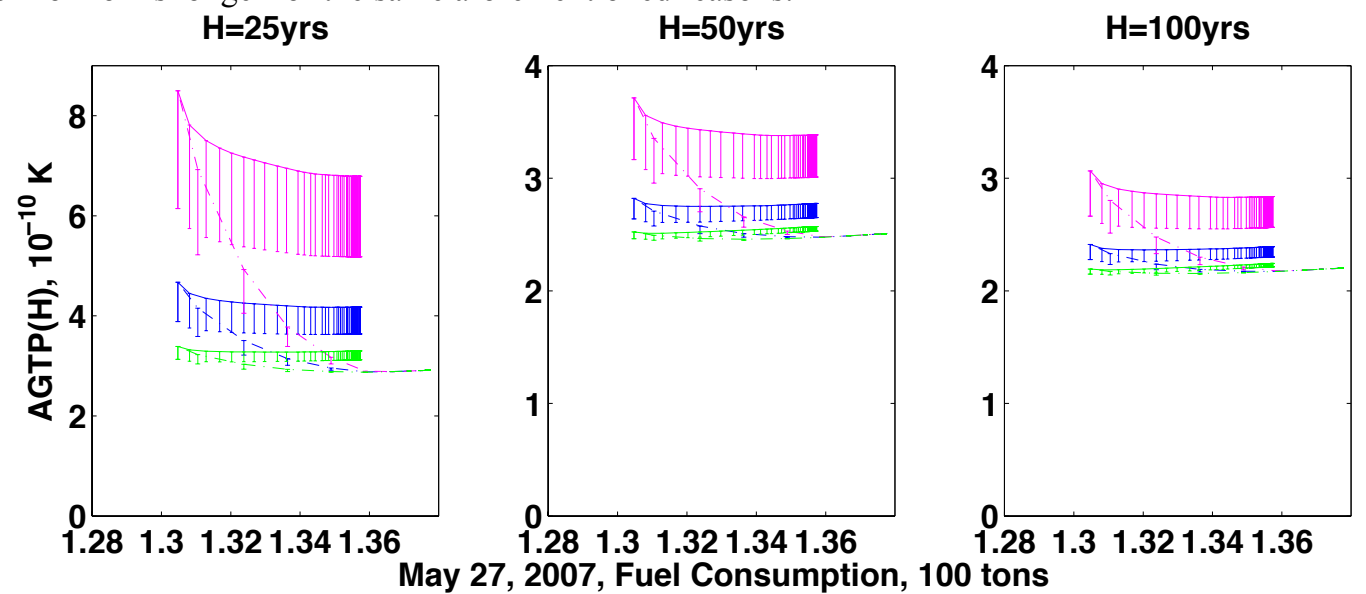

Figure 9. Total surface temperature change due to fuel burn and contrail formation for May 27, 2007.

Figure 10 and Figure 11 show the surface temperature change for a medium and low contrails day, respectively. The figures show that the reductions of surface temperature change are smaller for these days because contrails are less severe. This is particularly true when time horizon is longer where AGTP are mostly contributed by $\mathrm{CO}_{2}$ emissions only. The curves for May 24 are almost flat since contrails are very low. Flying wind-optimal should minimize climate change and the fuel cost. These curves can be used to develop optimal fleet allocation and scheduling strategies to minimize climate change for a given amount of additional fuel burn. 


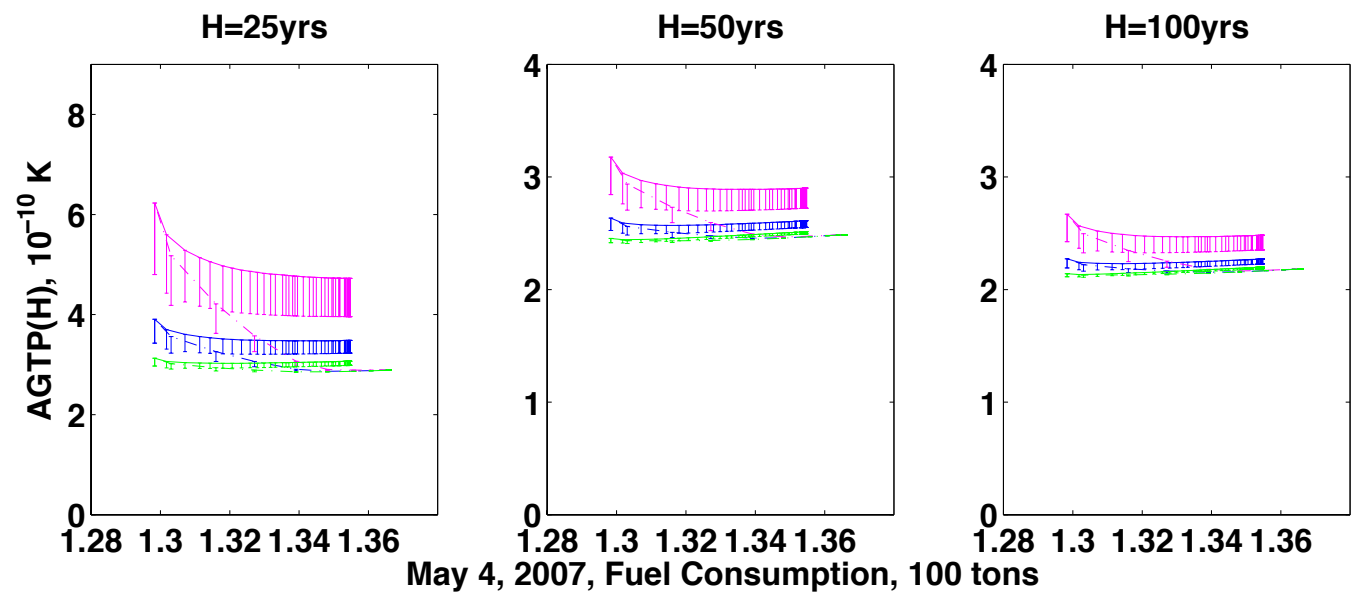

Figure 10. Total surface temperature change due to fuel burn contrails formation for May 4, 2007.
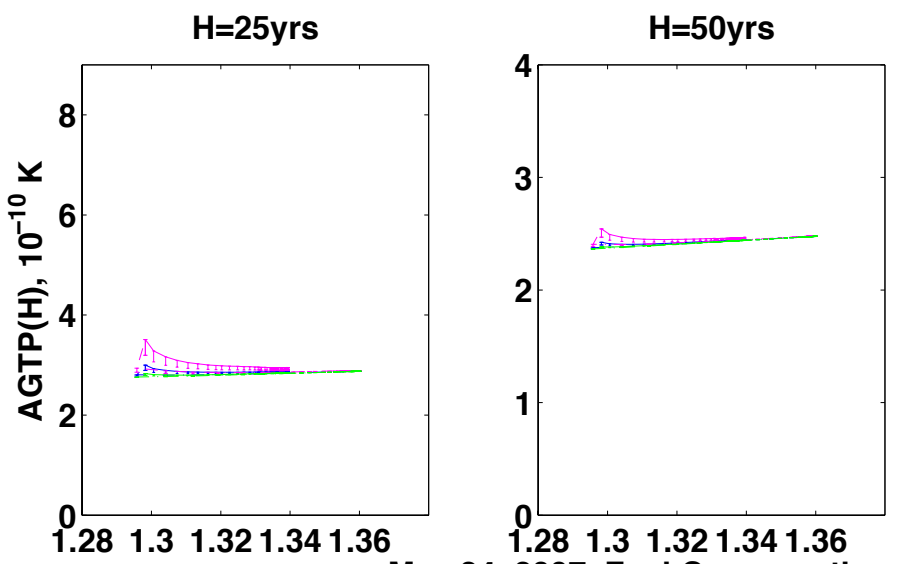

May 24, 2007, Fuel Consumption, 100 tons

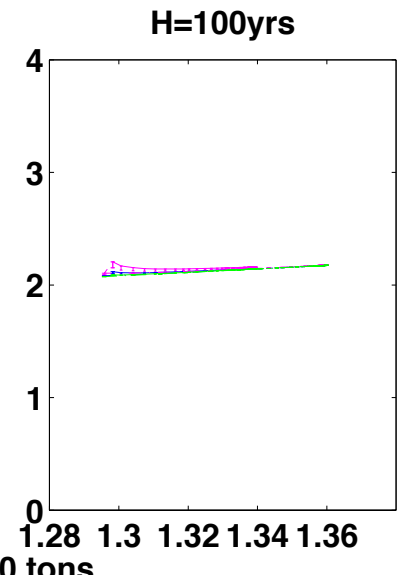

Figure 11. Total surface temperature change due to fuel burn contrails formation for May $24,2007$.

\section{Conclusion}

This paper described a simulation capability that can be used to evaluate technology and concepts to mitigate the impact of aviation emissions on climate. The capability brings together metrics useful in aviation operations with those used in climate studies. The capability can be used to make trade-offs between extra fuel cost and reduction in climate impact. When altitude is optimized during a severe contrails day, burning extra $5.4 \%, 4.6 \%$, and $4.6 \%$ fuel reduces the global surface temperature change by $66 \%, 38 \%$, and $15 \%$, respectively, for contrails radiative forcing of $30 \mathrm{Wm}^{-2}, 10 \mathrm{Wm}^{-2}$, and $3.3 \mathrm{Wm}^{-2}$ for a 25 -year time horizon. When 100 -year time horizon is considered, $4.6 \%$, $4.6 \%$, and $3.1 \%$ extra fuel reduces the absolute global temperature potentials by $29 \%, 10 \%$, and $1.8 \%$, respectively. Aircraft trajectories selected for minimum temperature changes are closer to wind-optimal because they minimize $\mathrm{CO}_{2}$ emission that has a life time much longer than contrails. This condition also prevails when contrails radiative forcing is smaller or a longer time horizon is considered. Similar trends are observed during the medium and low contrails days in which the reductions of surface temperature change are smaller because contrails formations are less severe. There is considerable uncertainty in our understanding of the radiative forcing associated with emissions and contrails. The parameters in the simulation can be used to evaluate the effect of various uncertainties in emission models and contrails. It can also be used to evaluate the impact of different decision horizons. Alternatively, the optimization results from the simulation can be used as inputs to other tools that monetize global climate impacts like the FAA's Aviation Environmental Portfolio Management Tool for Impacts.

\section{References}

${ }^{1}$ Meyn, L., Windhorst, R., Roth, K., Drei, D. V., Kubat, G., Manikonda, V., Roney, S. R. G., Huang, A., and Couluris, G., "Build 4 of the Airspace Concept Evaluation System," AIAA Modeling and Simulation Technologies Conference, AIAA-20066610, Keystone, Colorado, August 2006. 
${ }^{2}$ Erzberger, H., Davis, T. J., Green, S.M., "Design of Center-TRACON Automation System,” Proceedings of the AGARD Guidance and Control Panel $56^{\text {th }}$ Symposium on Machine Intelligence in Air-Traffic Management, Berlin, GDR, 1993, pp. 52-114.

${ }^{3}$ Bilimoria, K., Sridhar B., Chatterji, G., Sheth, K., and Grabbe, S. "FACET: future ATM concepts evaluation tool," Air Traffic Control Quarterly, Vol. 9, No. 1, 2001.

${ }^{4}$ IPCC, 1997, Greenhouse Gas Inventory Reference Manual, Intergovernmental Panel on Climate Change, Cambridge University Press, Cambridge, UK.

${ }^{5}$ Meerkötter, R., Schumann, U., Minnis, P., Doelling, D. R., Nakajima, T., and Tsushima, Y. "Radiative Forcing by Contrails," Ann. Geophysicae, Vol. 17, 1999, pp. 1080-1094, doi: 10.1007/s00585-999-1080-7.

${ }^{6}$ Fuglestvedt, J. S., Shine, K. P., Berntsen, T., Cook, J., Lee, D. S., Stenke, A., Skeie, R. B., Veldes, G. J. M., ad Waitz, I. A., "Transport impacts on Atmosphere and Climate: Metrics," Atmosphere Environment, Vol. 44, No. 37, 2010, pp. 4648-4677, doi:10.1016/j.atmosenv.2009.04.044.

${ }^{7}$ Khodayari, A., Wuebbles D. J., Olsen, S., Fuglestvedt, J.S., Berntsen, T., Lund, M. T., Waitz, I., Wolfe, P., and Forster, P., "Intercomparision and evaluation of the capabilities of simple climate models to project the effects of aviation on climate," WCRP Conference, October 2011.

${ }^{8}$ Aviation environmental Portfolio Management Tool, Partnership for AiR Transportation Noise \& Emissions reduction, http://web.mit.edu/aroastro/partner/apmt.

9"User Manual for the Base of Aircraft Data (BADA), Revision 3.6," Eurocontrol Experimental Center (EEC) Note No. 10/04, Project ACE-C-E2, Sept. 2004.

${ }^{10}$ Federal Aviation Administration, Washington, DC, Aviation Environmental Design Tool (AEDT) User Guide: Betalc, October 2010.

${ }^{11}$ Hadaller, O. J. and Momenthy, A. M., “The Characteristics of Future Fuels,” Project Report D6-54940, Boeing publication, 1989.

${ }^{12}$ Baughcum, S., Tritz, T., Henderson, S., and Pickett, D., "Scheduled Civil Aircraft Emission Inventories for 1992: Database Development and Analysis," Project Report NASA CR 4700, April 1996.

${ }^{13}$ Schumann, U., "On Conditions for Contrail Formation from Aircraft Exhausts," Meteorologische Zeitschrift, N. F. 5, Feb. 1996, pp. 4-23.

${ }^{14}$ Appleman, H., "The Formation of Exhaust Condensation Trails by Jet Aircraft," Bull. Amer. Meteor. Soc., Vol. 34, 1953, pp. 14-20.

${ }^{15}$ Ponater, M., Marquart, S., and Sausen, R., "Contrails in a Comprehensive Global Climate Model: Parameterization and Radiative Forcing Results,” Journal of Geophysical Research, Vol.107, Issue D13, 2002, pp. ACL 2-1.

${ }^{16}$ Sridhar, B., Ng, H. K., and Chen, N. Y., "Aircraft Trajectory Optimization and Contrails Avoidance in the Presence of Winds," Journal of Guidance, Control and Dynamics, Vol. 34, No. 5, September-October 2011, pp 1577-1583.

${ }^{17}$ Sorai, M., and Oshumi, T. "Ocean Uptake Potential for Carbon dioxide sequestration," Geochemical Journal, Vol. 39, 2005 , pp. 29-45.

${ }^{18}$ Fortunat Joos, I. Colin Prentice, Stephen Sitch, Robert Meyer, Georg Hooss, Gian-Kasper Plattner, Stefan Gerber, and Klaus Hasselmann, "Global warming feedbacks on terrestrial carbon uptake under the Intergovernmental Panel on Climate Change (IPCC) emission scenarios," Global Biogeochemical Cycles, Vol. 15, No. 4, Pp 891-907, December 2001.

${ }^{19}$ Sausen, R., and Schumann, U. "Estimate of the climate response of $\mathrm{CO}_{2}$ and $\mathrm{NO}_{\mathrm{x}}$ emissions scenarios," Climate Change, 44, pp 27-58, 2000.

${ }^{20}$ Hartmann, D.L., Global Physical Climatology, Academic Press, San Diego, CA, 1994.

${ }^{21}$ Boucher, O., and Reddy, M.S., "Climate trade-off between black carbon and carbon dioxide emissions," Energy Policy, 36, pp 193-200, 2008 .

${ }^{22}$ Wuebbles,D., Forster,P., Rogers, H., and Herman, R., "Issues and Uncertainties affecting Metrics for Aviation Impacts on Climate," Bulletin of American Meteorological Society, pp 491-495, April 2010.

${ }^{23}$ Shine, K. P., Fuglestvedt, J. S., Hailemariam, K., Stuber, N., 2005b. "Alternatives to the global warming potential for comparing climate impacts of emissions of greenhouse gases," Climatic Change 68, 281-302.

${ }^{24}$ Forster, P., Ramaswamy, V., Artaxo, P., Berntsen, T., Betts, R., Fahey, D.W., Haywood, J., Lean, J., Lowe, D.C., Myhre, G., Nganga, J., Prinn, R., Raga, G., Schulz, M., Van Dorland, R., 2007a. Changes in atmospheric constituents and in radiative forcing. In: Solomon, S., et al. (Eds.), Climate Change 2007: the Physical Science Basis. Contribution of Working Group I to the Fourth Assessment Report of the Intergovernmental Panel on Climate Change. Cambridge University Press, Cambridge.

${ }^{25}$ Haywood, J. M., Allan, R. P., Bornemann, J., Forster, P. M., Francis, P. N., Milton, S., Rädel, G., Rap, A., Shine, K. P., and Thorpe, R., "A Case Study of the Radiative Forcing of Persistent Contrails Evolving into Contrail-Induced Cirrus," Journal of Geophysical Research, Vol. 114, D24201, doi:10.1029/2009JD012650, 2009.

${ }^{26}$ Schumann, U., Graf, K., and Mannstein, H., "Potential to Reduce the Climate Impact of Aviation by Flight Level Changes," $3^{\text {rd }}$ AIAA Atmosphere Space Environments Conference, AIAA Paper 2011-3376, Honolulu, Hawaii, 2011.

${ }^{27}$ Ponater, M. "Distinctive Efficacies of the components contributing to total aviation climate impact," Proceedings of the 2nd International Conference on Transport, Atmosphere and Climate (TAC-2). 22-25 June 2009, DLR-Forschungsbericht 2010-10, Köln-Porz, Germany, ISSN 1434-8454. Aachen, Germany, and Maastricht, The Netherlands, 2009, pp. $227-232$.

28“Benefit Analysis and Report for Domestic Reduced Vertical Separation Minimum (DRVSM)," CDM/DRVSM Work Group Report, FAA Air Traffic Organization System Operations Services, Sept. 2005. 
${ }^{29}$ Minnis, P., Schumann, U., Doelling, D. R., Gierens, K., and Fahey, D. W. "Global distribution of contrail radiative forcing," Geophys. Res. Lett. Vol. 26, No. 13, 1999, pp. 1853 - 1856

${ }^{30}$ Sausen, R., Isaksen, I., Hauglustaine, D., Grewe, V., Lee, D. S., Myhre, G., Köhler, M. O., Pitari, G., Schumann, U., Stordal, F., and Zerefos, C. "Aviation radiative forcing in 2000: An update on IPCC (1999)," Meteorol. Z. Vol. 14, 2005, pp. 555 - 561, 10.1127/0941-2948/2005/0049.

${ }^{31}$ Burkhardt, U., and Kärcher, B. "Global radiative forcing from contrail cirrus," Nature Clim. Change Vol. 1, 2011, pp. 5458, DOI: 10.1038/NCLIMATE1068. 